\title{
SPECTRAL PROPERTIES OF PROJECTION SIGNALS IN 3-D TOMOGRAPHY
}

\author{
Yingbo Li", Anton Kummert ${ }^{*}$, Fritz Boschen ${ }^{*}$ and Hans Herzog ${ }^{* *}$ \\ *Faculty of Electrical, Information and Media Engineering, \\ University of Wuppertal, D-42097, Germany \\ Phone: +4920243919 61, Fax: +492024391959 \\ Email:yingbo@uni-wuppertal.de \\ ${ }^{* *}$ Institute of Medicine, Research Center Jülich, Germany \\ Email: h.herzog@fz-juelich.de
}

\begin{abstract}
In this paper the spectral properties of two different types of projection signals appearing in 3-D tomography are considered. In particular, the spectral behaviour of the 3 -D projection signal generated via a plane integral is analysed and its effective support region is figured out. On the basis of this result, an optimal interleaved sampling pattern is established. Compared to the traditional progressive sampling method, a reduction of sampling points by the factor 4 is achieved. Copyright (C) 2005 IFAC
\end{abstract}

Keywords: multidimensional systems, sampled signals, sampling rates, inverse problem, transformation.

\section{INTRODUCTION}

Tomography methods for image generation from projections extend the human ability to gain non invasive views of internal structures of objects for a wide range of physical applications, including X-ray computerized tomography, emission tomography, radio astronomy, electron microscopy and ultrasonic reflectivity tomography (Kak and Slaney, 1987). These methods are applicable when measured data, the so-called projection signals, represent certain forms of integrals of the spatial distribution of some physical quantity. For instance, the blood flow and metabolism of living organisms can be visualised by means of positron emission tomography (PET), where the measurement of coincidence between pairs of detectors approximates line integrals of the radioactive tracer distribution along lines of response (LOR). On basis of the reconstructed tracer distribution, malfunctions which are characteristic for diverse illnesses can be diagnosed (Pietrzyk, 1997).

Due to the complexity of the three-dimensional (3-D) reconstruction algorithm, the long reconstruction time duration and the large size of projection data, the reconstruction of a 3-D distribution used to be simplified as a series of two-dimensional (2-D) reconstruction problems. A 3-D volume object had to be decomposed into a set of parallel transverse planes at first. With the data for each cross-section plane being acquired and reconstructed independently of any other plane, the mathematical and computational complexity could be considerably reduced, as well as the reconstruction effort. Finally the consecutively reconstructed planes could be stacked together to form the 3-D volume object. However, with the restriction caused by such 2-D data acquisition and 2$\mathrm{D}$ reconstruction methods, only the measurements lying within the transaxial planes could be exploited, which consequently led to a system sensitivity loss. Owing to the rapid progress in hardware in recent years, nowadays this bottle-neck effect in consequence of enormous computa-tional demand can be efficiently circumvented. By using suitable true 3-D reconstruction algorithms and fully 3-D data acquisition or increased axial field of view, not only the transaxial, but also the oblique measurements can 
be taken into account. Thus, lower statistical noise in the reconstructed images can be achieved (Bendriem and Townsend, 1998).

The Radon transform and its 3-D extension are the underlying fundamental concept used for 2-D and 3D tomography. Via Radon transform the projection signals and the image object are related. However, in practical applications of reconstruction from projections, the measurements correspond only to estimated samples of the underlying continuous projection data. In order to acquire unadulterated projection data, sampling requirements for the measurement must be imposed.

In the present paper the spectral properties of projection signals in 3-D tomography with respect to frequency band limitations are analysed. Based on these analyses an optimal sampling pattern for projection data in 3-D tomography is proposed. The approach presented here involves a merely 3-D viewpoint and enlightens the problem from another respect.

It is shown that an optimal interleaved sampling grid in 3-D space requires only one-fourth of sampling points compared to the commonly used progressive sampling grids. In the next section, extension of the Radon transform for 3-D tomography is introduced, in particular the new concept of projection data which represents integrals over planes through the volume distribution. In section 3 the 3 -D spectral behaviour of projection data collected as planeintegrals will be evaluated. The resulting special type of sampling pattern for projection data will finally be proposed in section 4 .

\section{RADON TRANSFORM USED IN 3-D TOMOGRAPHY}

For the purpose of continuity, the usage of the Radon transform in 2-D tomography will be briefly reviewed at first (Herman, et al., 1990). With a distribution function (image) $f(x, y)$ defined on $\mathbb{R}^{2}$, which represents a spatial distribution of some physical quantity in two dimensions, the 2-D Radon transform of this distribution function, denoted as $p(\vartheta, u)$, is traditionally defined as the line integral of $f$ along a line perpendicular to the $u$-axis at an angle $\vartheta$ and at distance $u$ from the origin. This is illustrated by Figure 1, where $x, y$ are the Cartesian coordinates. Mathematically, the coordinate $u$ for angle $\vartheta$ can be denoted as a function of the variables $x$ and $y$ in the following way:

$$
u=x \cos \vartheta+y \sin \vartheta .
$$

Thereby the projection $p(\vartheta, u)$ can be defined as

$$
p(\vartheta, u)=\int_{-\infty}^{+\infty} \int_{-\infty}^{+\infty} f(x, y) \cdot \delta(u-x \cos \vartheta-y \sin \vartheta) d x d y,
$$

where $\delta(x)$ is the Dirac impulse function.
In addition, the well-known 2-D Fourier slice theorem relates the Fourier transform (with respect to variable $u$ ) of the projection data with the Fourier transform of distribution function $f(x, y)$ in the frequency domain. It says that the one-dimensional Fourier transform with respect to $u$ of the projection signal $p(\vartheta, u)$ at the angle $\vartheta$ is equal to a central slice of the 2-D Fourier transform of the distribution function $f(x, y)$ at the same angle $\vartheta$.

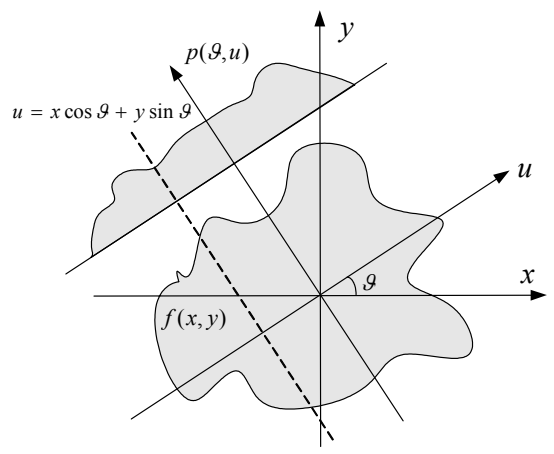

Fig. 1. Principles of the 2-D Radon transform

With the definitions above as background, the 3-D Radon transform and its related 3-D Fourier slice theorem can be deduced straightforward by means of generalization of its 2-D counterparts. However, due to miscellaneous physical natures of applications, the projection data may be accomplished by varying form of integrals. In the following two sub-sections, two different types of projections are introduced.

\subsection{Projection data as integrals over lines}

Consistent with the 2-D definition of projection as line integral, 3-D projections are conventionally treated as 2-D sets of parallel line-integrals in 3-D space. With a distribution $f(x, y, z)$ defined on $\mathbb{R}^{3}$, its 3-D Radon transform on basis of line-integration as projection data, will be discussed at first. The projection signal $p(u, v, \vartheta, \varphi)$ can be defined as the line integral of $f$ along a line orthogonal to a plane with coordinates $(u, v)$. This plane has an azimuthal angle $\varphi$ and a co-polar angle $\vartheta$. The integration line crosses this plane at distance $u$ and $v$ from the reference axes, which is illustrated in Figure 2. $(u, v, t)$ is the rotated coordinate system given by

$$
\left[\begin{array}{l}
u \\
v \\
t
\end{array}\right]=\left[\begin{array}{ccc}
-\sin \varphi & \cos \varphi & 0 \\
-\cos \varphi \sin \vartheta & -\sin \varphi \sin \vartheta & \cos \vartheta \\
\cos \varphi \cos \vartheta & \sin \varphi \cos \vartheta & \sin \vartheta
\end{array}\right] \cdot\left[\begin{array}{c}
x \\
y \\
z
\end{array}\right] .
$$

Mathematically, the line of integration in 3-D space can be denoted with two functions of the variables $\vartheta, \varphi, u$ and $v$ in the following way:

$$
\left\{\begin{array}{l}
u=-x \sin \varphi+y \cos \varphi \\
v=-x \cos \varphi \sin \vartheta-y \sin \varphi \sin \theta+z \cos \vartheta
\end{array}\right.
$$

Thereby the projection $p(u, v, \vartheta, \varphi)$ can be defined as 


$$
\begin{array}{r}
p(u, v, \vartheta, \varphi)=\int_{-\infty}^{+\infty} \int_{-\infty}^{+\infty} \int_{-\infty}^{+\infty} f(x, y, z) \cdot \delta(u+x \sin \varphi-y \cos \varphi) \\
\cdot \delta(v+x \cos \varphi \sin \vartheta+y \sin \varphi \sin \vartheta-z \cos \vartheta) d x d y d z .
\end{array}
$$

It should be noticed that the projection data resulting from line integrals in 3-D space are four dimensional in nature, which indirectly implies the existence of redundant information contained in the projection data. Nevertheless, in practical applications, adequate exploitation of the additional information is a commonly used method to improve the sensitivity of the measurement system. For instance, the key point of the 3-D PET imaging system is that the collection of the seemingly redundant information along the oblique LORs, if used properly, can improve reconstructed image SNR by reducing statistical noise (Bendriem and Townsend, 1998).

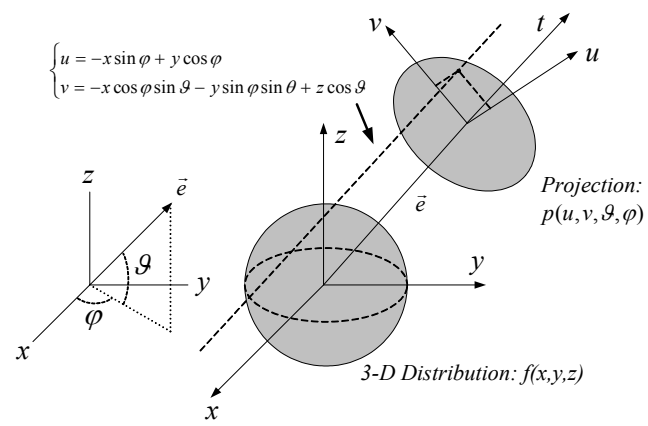

Fig. 2. Principles of the 3-D Radon transform with line integration as projection data

The extended 3-D Fourier slice theorem associated with projection data as line integrals states that the 2D Fourier transform of the projection data $p(u, v, \vartheta, \varphi)$ with respect to $u$ and $v$ at the angle $\vartheta$ and $\varphi$ corresponds to a central plane of the 3-D Fourier transform of the distribution $f(x, y, z)$ at the same spatial angle. This is illustrated in figure 3, where the frequency coordinates in the object and projection spaces are subject to the same rotational relations as between the spatial coordinates, defined in (3).

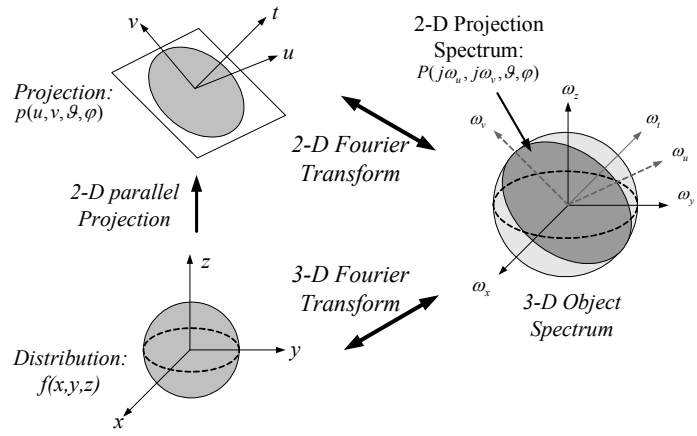

Fig. 3. Illustration of the extended 3-D Fourier slice theorem associated with projection data as line integrals

\subsection{Projection data as integrals over planes}

Instead of generating the projection data as line integrals, projections in the 3-D case can be alternatively accomplished by integrations over planes. From this viewpoint, the 3-D Radon transform can be newly discussed in an alternative way. With a distribution $f(x, y, z)$ defined on $\mathbb{R}^{3}$, whose 3-D Radon transform is denoted as $p(t, \vartheta, \varphi)$, the projections can be defined as integration of $f$ on a plane, whose orientation is described by a unit vector with two angular designations, $\vartheta$ and $\varphi$. The distance of plane from the origin is depicted by the variable $t$. In figure 4 this situation is illustrated. The rotated coordinate system $(u, v, t)$ is given by equation (3) again. Geometrically, the 3-D Radon transform defined in this manner maps a spatial distribution into the set of its plane integrals in 3-D space. Each point in the projection domain $(t, \vartheta, \varphi)$ corresponds to a plane in the spatial domain $(x, y, z)$. The plane, on which integration occurs, can be mathematically described as follows:

$$
t=x \cos \vartheta \cos \varphi+y \cos \vartheta \sin \varphi+z \sin \vartheta,
$$

so that the projection $p(t, \vartheta, \varphi)$ can be defined as

$$
\begin{aligned}
& p(t, \vartheta, \varphi)=\int_{-\infty}^{+\infty} \int_{-\infty}^{+\infty} \int f(x, y, z) . \\
& \delta(t-x \cos \vartheta \cos \varphi-y \cos \vartheta \sin \varphi-z \sin \vartheta) d x d y d z .
\end{aligned}
$$

Projection data as plane-integrations is also of interest in various practical applications. If we consider for example a pulse emitting echo sounder in sonar tomography (far field), then the received sonar signal at a certain time instance is the integral over all scatters on a plane at a certain distance. This distance is associated with the time instance via the propagation velocity of sound waves in water.
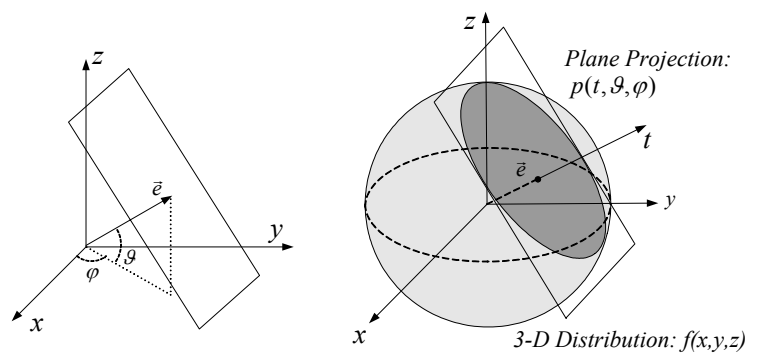

Fig. 4. Principles of the 3-D Radon transform with plane integration as projection data

In accordance with the new definition, the 3-D Fourier slice theorem also changes its meaning. It says respectively that the one-dimensional Fourier transform with respect to $t$ of the plane projection $p(t, \vartheta, \varphi)$ at the spatial angles $\vartheta$ and $\varphi$ is equal to the 3-D Fourier transform of the distribution function $f(x, y, z)$ along a central line at the same spatial angles.

\section{SPECTRAL PROPERTIES OF PROJECTION DATA IN CASE OF PLANE INTEGRALS}

Both of the two 3-D Radon transform types 
mentioned in section 2 apply to continuous signal representations. In practical applications, however, measurements are available as estimated samples. Hence an adequate arrangement of the measurement equipment is the key to achieve projection data in accordance with the sampling theorem, which means that an optimal sampling pattern for the projection signal has to be established.

For this purpose, the basic support region of the projection in the frequency domain (spectrum) should be investigated. The sampling grid can then be obtained in filling the frequency space with this basic shape. A similar approach in 2-D case has been used in (Rattey et al., 1981; Boschen, 2000). While such an approach is absolutely meaningful for the analysis of the projection data in case of plane integrals, it imposes certain problems for the projection data in case of line integrals. Since the projection data of a 3-D distribution as line integrals is of four-dimensional nature, it is impossible to establish the sampling grid using the "fill-and-see" method. Furthermore, due to the redundant information contained in the projection data, it makes the study of the spectral properties of the projection data more complicated. In view of these facts, the analysis of the spectral properties of the projection signals in this paper is restricted to the case described in sub-section 2.2 as plane integrals.

Since the 3-D distribution of physical quantities in practical applications can be always assumed to have a limited spatial extent, the 3-D distribution function to be treated here can also be seen as spatially bounded. In addition, due to the well-known fact that any 3-D function can be decomposed as continuous sum (integral) of weighted impulse functions, i.e.,

$$
\begin{aligned}
& f(x, y, z)=\int_{-\infty}^{+\infty} \int_{-\infty}^{+\infty} \int f\left(x_{0}, y_{0}, z_{0}\right) \\
& \quad \cdot \delta\left(x-x_{0}, y-y_{0}, z-z_{0}\right) d x_{0} d y_{0} d z_{0},
\end{aligned}
$$

it is self-evident firstly to consider the spectral behaviour of the projection data caused by a simple impulse function $\delta\left(x-x_{0}, y-y_{0}, z-z_{0}\right)$. Substituting this impulse function into (7) directly yields

$$
\begin{aligned}
& p(t, \vartheta, \varphi)= \\
& \quad \delta\left(t-x_{0} \cos \vartheta \cos \varphi-y_{0} \cos \vartheta \sin \varphi-z_{0} \sin \vartheta\right)
\end{aligned}
$$

Because of the linear nature of the extended 3-D Radon transform explained in sub-section 2.2 and equation (8), the 3 -D projection of $f(x, y, z)$ can then be viewed as superposition of these impulse functions of form defined in (9) with varying $x_{0}$, $y_{0}$, and $z_{0}$. From this point of view, the feasibility to determine the effective band-region of the projection spectrum of $f(x, y, z)$ is provided by means of analysing the spectral behaviour of an individual impulse function. The optimal sampling pattern can then be simply determined by observing how the 3-D frequency domain is covered by the periodic replication of the basic spectral support region of the impulse function of form (9).

The 1-D Fourier transform of the impulse function of (9) with respect to variable $t$ is given by

$$
\begin{array}{r}
F_{p}\left(\omega_{t}, \vartheta, \varphi\right)=\int_{-\infty}^{+\infty} p(t, \varphi, \vartheta) e^{-j \omega_{t} t} d t \\
=e^{-j \omega_{t}\left(x_{0} \cos \vartheta \cos \varphi+y_{0} \cos \vartheta \sin \varphi+z_{0} \sin \vartheta\right)} \\
=e^{-j \omega_{t} r_{0}\left[\cos \theta_{0} \cos \vartheta \cos \left(\varphi-\phi_{0}\right)+\sin \theta_{0} \sin \vartheta\right]},
\end{array}
$$

by using the following variable representation

$$
\left\{\begin{array}{l}
x_{0}=r_{0} \cos \theta_{0} \cos \phi_{0} \\
y_{0}=r_{0} \cos \theta_{0} \sin \phi_{0} . \\
z_{0}=r_{0} \sin \theta_{0}
\end{array}\right.
$$

It is obvious that this 1-D Fourier transform is periodic in $\vartheta$ and $\varphi$, i.e.,

$$
\begin{aligned}
& F_{p}\left(\omega_{t}, \vartheta, \varphi\right)=F_{p}\left(\omega_{t}, \vartheta+2 m \pi, \varphi\right) \\
& F_{p}\left(\omega_{t}, \vartheta, \varphi\right)=F_{p}\left(\omega_{t}, \vartheta, \varphi+2 n \pi\right) .
\end{aligned}
$$

Consequently, the complete 3-D Fourier transform, i.e. with respect to $t, \vartheta$, and $\varphi$, is of discrete nature in the $\omega_{\vartheta}$ - and $\omega_{\varphi}$-direction and can be written as

$$
\begin{aligned}
& F_{p}\left(\omega_{t}, \omega_{\vartheta}, \omega_{\varphi}\right)= \\
& 4 \pi \sum_{m=-\infty}^{+\infty} \sum_{n=-\infty}^{+\infty} e^{-j n\left(\phi_{0}+\frac{\pi}{2}\right)} \cdot \delta\left(\omega_{\vartheta}-m\right) \cdot \delta\left(\omega_{\varphi}-n\right) \\
& \cdot \int_{0}^{\pi} \cos \left(\omega_{t} r_{0} \sin \theta_{0} \sin \vartheta+m \vartheta\right) \cdot J\left(n, \omega_{t} r_{0} \cos \theta_{0} \cos \vartheta\right) d \vartheta,
\end{aligned}
$$

where

$$
\begin{aligned}
& J\left(n, \omega_{t} r_{0} \cos \theta_{0} \cos \vartheta\right)= \\
& \frac{1}{2 \pi} \int_{-\pi}^{+\pi} e^{j\left(n \tau-\omega_{t} r_{0} \cos \theta_{0} \cos \vartheta \sin \tau\right)} d \tau
\end{aligned}
$$

is the $n$ th-order Bessel function of the first kind.

Since the integral in (13) cannot be solved explicitly, numerical analysis method has to be used to figure out the shape of the effective support region. While the projection spectrum is of complex nature, the analysis focuses only on the magnitude of the spectrum to assess its spectral extent in frequency domain. In figure 5 the numerical results of an exemplary impulse function without phase factor is displayed. Due to the difficulty in displaying trivariate functions, the magnitudes of the projection spectrum are illustrated in dependence of the variable $\omega_{t}$. It is easy to recognize that the magnitude is of discrete nature in $\omega_{\vartheta}$ - and $\omega_{\varphi}$-direction. In $\omega_{t}$ direction, the function is continuous. 
An examination of the computational results reveals that the effective support region of the projection spectrum in frequency domain resembles a shape of head-to-head adhered double-cone of infiniteextension as displayed in figure 6(b). The amplitude has its maximum exactly in the centre, i.e., at $\omega_{t}=0$.

With the increase of $\omega_{t}$, the amplitude begins to decrease. Simultaneously, the effective region with respect to $\omega_{\vartheta}$ and $\omega_{\varphi}$ becomes larger. Defining the band region of $F_{p}\left(\omega_{t}, \omega_{\vartheta}, \omega_{\varphi}\right)$ with respect to the discrete $\omega_{\vartheta}$ - and $\omega_{\varphi}$-direction for a given $\omega_{t}$ as the number of terms, which are necessary to include $98 \%$ of the whole energy, the following relation turns out for the band-width in $\omega_{\vartheta}{ }^{-}$and $\omega_{\varphi^{-}}$ direction

$$
\Delta \omega_{\vartheta, \varphi}>R_{M}\left|\omega_{t}\right|+1,
$$

where $R_{M}$ is defined as the spatial bound of the 3-D distribution function. Due to the discrete nature of $\omega_{\vartheta}$ and $\omega_{\varphi}$, this relation can be further modified to

$$
\Delta \omega_{\vartheta, \varphi}>\left[R_{M}\left|\omega_{t}\right|\right]+1,
$$

where the bracket indicates that the largest integer value smaller than $R_{M}\left|\omega_{t}\right|$ should be used.

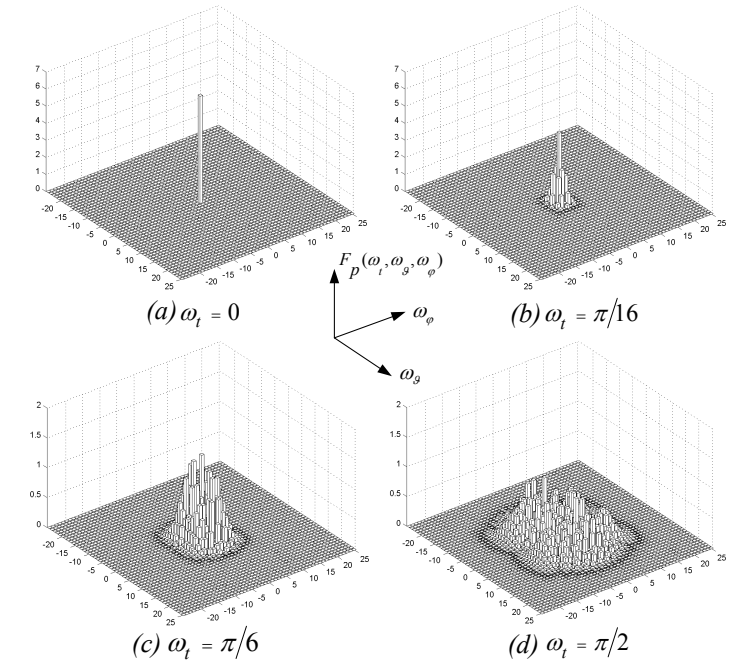

Fig. 5. Illustration of numerical results of an exemplary impulse function without phase factor. The outcomes are displayed subject to the variable $\omega_{t}$.

For a spatially limited function shown in figure 6(a), the effective support region of projection spectrum is displayed in figure 6(b). Known from signal theory that a function of finite spatial support has a nonband-limited spectrum, the head-to-head adhered double-cone also has no band limitation with respect to the $\omega_{t}$-direction. However, in practice the spatiallimited distribution often can be treated as approximately band limited in the frequency domain.
Based on this assumption, $F_{p}\left(\omega_{t}, \omega_{\vartheta}, \omega_{\varphi}\right)$ can be considered as quasi zero for the region $\left|\omega_{t}\right|>\omega_{M}$ with $\omega_{M}$ as desired bound approximation, and hence the support region of the projection spectrum has a shape of two head-to-head adhered double-cone of finite-length $\omega_{M}$, which is shown in 6(d).

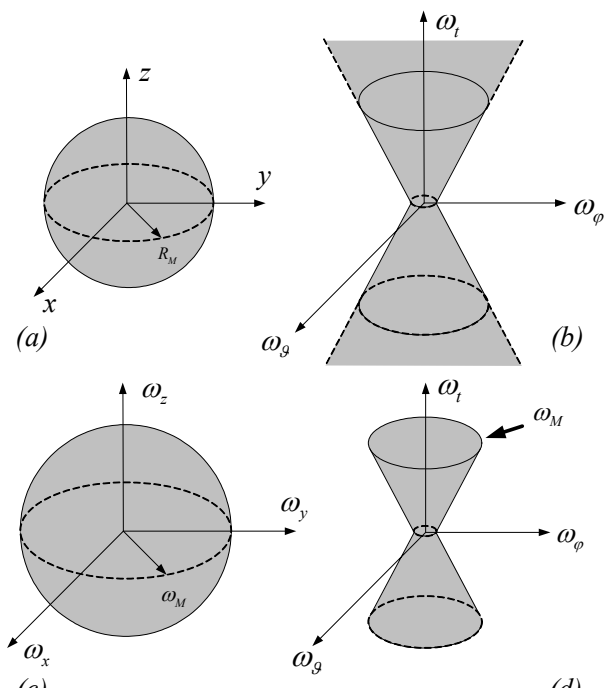

(c)

(d)

Fig. 6. Illustration of various support regions in spatial and frequency domains. (a) spatial-limited 3D distribution; (b) projection spectrum with nonbandlimited shape; (c) bounded spectrum in $\left(\omega_{x}, \omega_{y}, \omega_{z}\right)$ domain; (d) projection spectrum with band-limited shape

\section{OPTIMAL SAMPLING PATTERN FOR PROJECTION DATA}

Owing to the acquired basic shape of the projection spectrum as head-to-head adhered double-cone of finite-length, an optimal sampling pattern can be obtained to improve efficiency. This occurs in observing how the 3-D frequency space can be optimally covered by the periodic replication of the double-cones. It can be proved that merely about one-fourth of samples compared to originally needed samples are really required to avoid the adulteration of the projection data.

Traditionally, the projection data is progressively sampled on a rectangular grid. This scheme is illustrated in figure 7. Correspondingly, the sampling matrix is to be defined straightforward as

$$
\Omega=\left(\begin{array}{ccc}
\Omega_{t} & 0 & 0 \\
0 & \Omega_{\vartheta} & 0 \\
0 & 0 & \Omega_{\varphi}
\end{array}\right) .
$$

Due to the acquired knowledge about the basic shape of the projection spectrum, the values of individual elements in the sampling matrix can be determined as $\Omega_{t}=2 \omega_{M}, \Omega_{\vartheta}=\Omega_{\varphi}=2\left(\left[R_{M}\left|\omega_{M}\right|\right]+1\right)$. With the 
relation between the sampling matrix $\Omega$ and the period matrix $T$

$$
\Omega^{t}=2 \pi T^{-1},
$$

the period matrix can be calculated as

$$
\begin{gathered}
T=\left(\begin{array}{ccc}
T_{t} & 0 & 0 \\
0 & T_{\vartheta} & 0 \\
0 & 0 & T_{\varphi}
\end{array}\right)= \\
\left(\begin{array}{ccc}
\pi / \omega_{M} & 0 & 0 \\
0 & \pi /\left(\left[R_{M}\left|\omega_{M}\right|\right]+1\right) & 0 \\
0 & 0 & \pi /\left(\left[R_{M}\left|\omega_{M}\right|\right]+1\right)
\end{array}\right) .
\end{gathered}
$$

Consideration of figure 7 indicates apparently that the 3-D frequency space is not optimally filled by progressive sampling procedure. The gaps between the double-cones denote lavishness in the frequency domain. On the basis of the double-cone shape, an interleaving sampling pattern can be utilized to eliminate the gaps and to increase the information content. In figure 8 such an interleaved sampling pattern is illustrated.

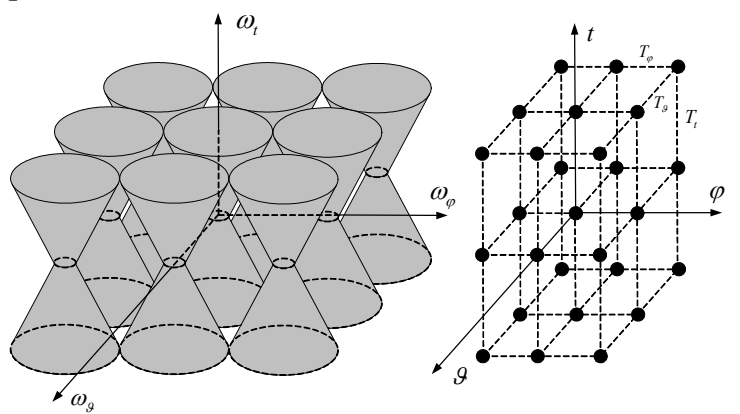

Fig. 7. Schematic description of the progressive sampling on the rectangular grid

According to the illustration in figure 8 , the new optimal sampling matrix can be depicted as

$$
\Omega_{o p t}=\left(\begin{array}{ccc}
\Omega_{t} / 2 & \Omega_{t} / 2 & \Omega_{t} / 2 \\
\Omega_{\vartheta} / 2 & -\Omega_{\vartheta} / 2 & 0 \\
0 & 0 & \Omega_{\varphi} / 2
\end{array}\right) .
$$

Using the relation defined in (18), the new period matrix is correspondingly determined as

$$
T_{o p t}=\left(\begin{array}{ccc}
T_{t} & T_{t} & 0 \\
-T_{\vartheta} & T_{\vartheta} & 0 \\
-T_{\varphi} & -T_{\varphi} & 2 T_{\varphi}
\end{array}\right)
$$

with $T_{t}, T_{\vartheta}$ and $T_{\varphi}$ calculated in (19).

Comparing the support point grid in figure 7 and the optimized support point grid in figure 8 , it turns out that only one-fourth of the original points are needed. The individual double-cones do not touch each other, so that no aliasing arise. Therefore the projection signals can be adequately represented by the samples.

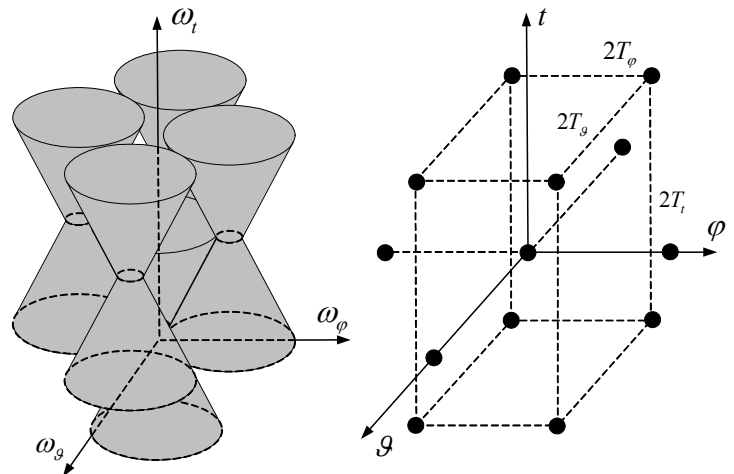

Fig. 8. Schematic description of the interleaved sampling pattern

\section{CONCLUSIONS AND FUTURE WORK}

In this paper, the spectral behaviour of 3-D projection signals, especially in the case of plane integrals, in 3-D tomography is analysed. The approach involves exclusively a 3-D point of view. With the acquired knowledge that the band region of the projection spectrum is a head-to-head adhered double-cone of finite-length, a new optimized sampling pattern is proposed. This sampling pattern reduces the number of sampling points by the factor 4 compared to the rectangular grid.

Since the projection spectrum of a function of finite spatial extent is nonband-limited in the frequency domain in an exact sense, the effective band-width has to be considered. By analysing spatially nonlimited distributions, sharp band-limitations in the frequency domain can be achieved. However, detailed discussions of such an approach are very complex and beyond the scope of this paper.

\section{REFERENCES}

Bendriem, B. and D.W. Townsend (1991). The theory and practice of $3 D$ PET, Kluwer Academic publishers, Netherlands.

Boschen, F., (2000). Analysis and improvement of different image reconstruction and filtering methods, Logos Verlag, Berlin.

Kak, A.C. and M. Slaney (1987). Principles of computerized tomographic imaging, IEEE Press, New York.

Herman, G.T., A.K. Louis and F. Natterer (1990). Mathematical methods in tomography, Springer Verlag, Germany.

Pietrzyk, U. (1997). Positron emission tomography Physical background and applications, Shaker Verlag, Aachen.

Rattey, P.A. and A. G. Lindgren (1981). Sampling the 2-D Radon transform. IEEE transactions on acoustics, speech and signal processing, Vol. ASSP-29, No. 5, 994-1002. 